\title{
Studies in the atmospheric monitoring at the Pierre Auger Observatory using the upgraded Central Laser Facility
}

\author{
Carlos Medina-Hernandez ${ }^{* a}$ for Pierre Auger Collaboration ${ }^{b}$ \\ ${ }^{a}$ Department of Physics, Colorado School of Mines, Golden CO, USA. \\ ${ }^{b}$ Observatorio Pierre Auger, Av. San Martín Norte 304, 5613 Malargüe, Argentina \\ E-mail: auger_spokespersons@fnal.gov \\ Full author list: http://www.auger.org/archive/authors_2015_06.html
}

\begin{abstract}
The Fluorescence Detector (FD) at the Pierre Auger Observatory measures the intensity of the scattered light from laser tracks generated by the Central Laser Facility (CLF) and the eXtreme Laser Facility (XLF) to monitor and estimate the vertical aerosol optical depth $(\tau(\mathrm{z}, \mathrm{t}))$. This measurement is needed to obtain unbiased and reliable FD measurements of the arrival direction and energy of the primary cosmic ray, and the depth of the maximum shower development. The CLF was upgraded substantially in 2013 with the addition of a solid state laser, new generation GPS, a robotic beam calibration system, better thermal and dust isolation, and improved software. The upgrade also included a back-scatter Raman LIDAR to measure $\tau(\mathrm{z}, \mathrm{t})$. The new features and applications of the upgraded instrument are described. These include the laser energy calibration and the atmospheric monitoring measurements. The first $\tau(\mathrm{z}, \mathrm{t})$ results and comparisons after the upgrade are presented using different methods. The first method compares the FD hourly response to the scattered light from the CLF (or XLF) against a reference hourly profile measured during a clear night where zero aerosol contents are assumed. The second method simulates FD responses with different atmospheric parameters and selects the parameters that provide the best fit to the actual FD response. A third method uses the new Raman LIDAR receiver in-situ to measure the back-scatter light from the CLF laser. The results show a good data agreement for the first and second methods using FD stations located at the same distance from the facilities. Preliminary results of $\tau(\mathrm{z}, \mathrm{t})$ using the Raman LIDAR are presented as well.
\end{abstract}

The 34th International Cosmic Ray Conference,

30 July- 6 August, 2015

The Hague, The Netherlands

${ }^{*}$ Speaker. 


\section{Introduction}

The Pierre Auger Observatory, located in Malargüe, Argentina, explores the nature and origin of Ultra High Energy Cosmic Rays (UHECRs) with energies above $10^{18} \mathrm{eV}$ [1]. The observatory (Figure 1) combines a Fluorescence Detector (FD) and a Surface Detector array (SD) in a hybrid technique. The FD consists of 27 telescopes arranged at four sites along the perimeter of the observatory [2]. The SD is a collection of 1660 water-Cherenkov detectors, placed on a triangular grid with a separation of $1.5 \mathrm{~km}$ and covering an area of $3000 \mathrm{~km}^{2}$. Two laser facilities are located near the center of the SD array. Each facility is nearly equidistant from three of the four FD stations.

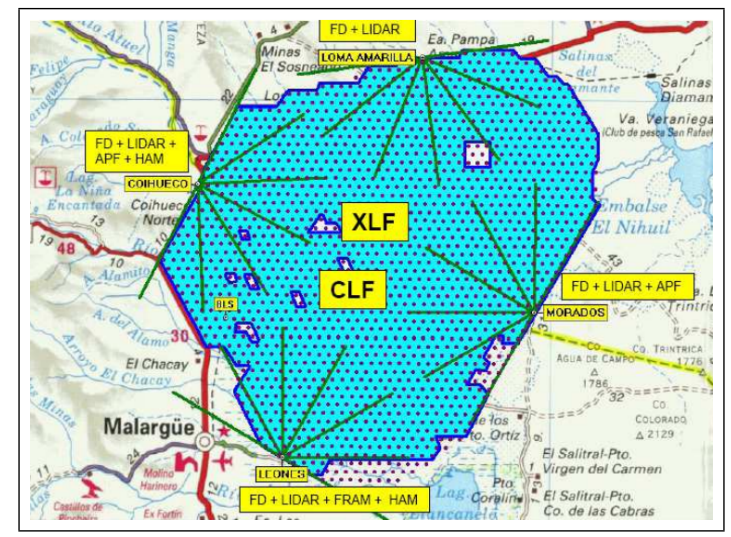

Figure 1: The configuration of the Pierre Auger Observatory.

UHECRs entering the atmosphere induce Extensive Air Showers (EASs). The FD uses the atmosphere as a giant calorimeter in which an EAS deposits most of its energy. The reliability of the reconstruction of any EAS requires an accurate description of the atmospheric conditions at the time of detection. The atmospheric description includes the measurement of the vertical aerosol optical depth for different heights and times $\tau(\mathrm{z}, \mathrm{t})$ during FD operations. For this purpose, the FD records tracks from the laser pulses generated by the Central Laser Facility (CLF) and the eXtreme Laser Facility (XLF). The CLF and XLF have been operational since 2004 and 2008, respectively. In early 2013, the CLF was upgraded with a Raman LIDAR receiver that measures $\tau(\mathrm{z}, \mathrm{t})$ independently.

\section{The CLF and XLF}

The CLF and XLF systems [3] generate $7 \mathrm{~ns}$ laser pulses at a nominal energy of $5 \mathrm{~mJ}$ and $355 \mathrm{~nm}$ wavelength, near the prominent $357 \mathrm{~nm}$ band of ultraviolet fluorescence in the air [4]. The laser tracks are distinguished from regular air shower events, via GPS timing identification. For atmospheric monitoring during FD operations, the CLF and XLF fire sets of 50 laser pulses, every 15 minutes, in the vertical direction. Other laser pulses are also fired between the atmospheric laser sets. For example, steered shots are directed toward astronomical objects of interest. The FD response to the scattered light from the fixed vertical laser-pulses at each FD site can be compared either with similar measurements recorded during a night free of aerosols, or with simulations of data under different aerosols conditions. These comparisons are used to calculate $\tau(\mathrm{z}, \mathrm{t})$. 
Both laser facilities are controlled by Single Board Computers (SBC) that can be operated and programmed remotely. These facilities are powered by solar panels that charge a battery bank. Local data produced during a run are transferred daily to a remote server.

\subsection{The upgraded CLF}

After nearly a decade of service, the CLF [5] was substantially upgraded during the first half of 2013 (Figure 2):

- A back-scatter Raman LIDAR receiver was installed.

- The original flash lamp pumped laser was replaced by a solid state laser.

- A newer GPS clock system improves the timing resolution from 100 to $20 \mathrm{~ns}$.

- The original $20 \mathrm{ft}$ shipping container was replaced by a newer $40 \mathrm{ft}$ unit with tighter doors and better insulation. A 2000 liter thermal reservoir coupled to the optical table was added to reduce thermal variations.

- The better sealed container features a separate room for the laser system to reduce the dust accumulation. This is important because dust accumulation on optical components increases the systematic uncertainty of laser energy delivered into the sky.

- A robotic system for automatic energy and polarization calibrations was added.
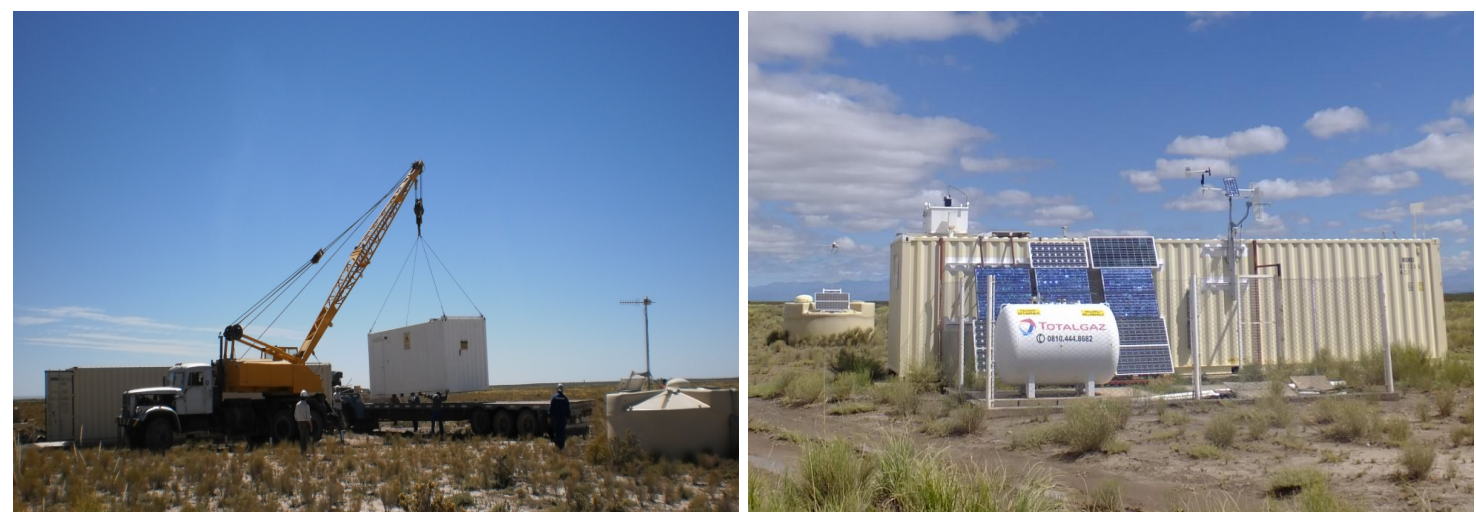

Figure 2: (Left) Replacing the old CLF container. (Right) The CLF after the upgrade was completed.

\subsection{The energy + polarization calibration system}

The relative energy of every laser shot is measured by a monitoring energy probe that collects a small percentage of the total laser shot energy $\left(E_{\text {monitoring }}\right)$. A second probe is temporarily positioned over the beam, for absolute energy calibrations $\left(E_{\text {calibration }}\right)$. A Calibration Factor $(C F)$ is calculated by averaging the ratio of the calibration and monitoring probes for 13 shots:

$$
C F=\frac{1}{13} \cdot \sum_{i=1}^{13}\left(\frac{E_{\text {calibration }}}{E_{\text {monitoring }}}\right)
$$


The calibration system provides $C F$ measurements as frequently as needed for vertical and steered modes (Figure 3). The system also includes a second energy probe for cross calibrations. An identical calibration system was installed in the XLF at the time of its construction.
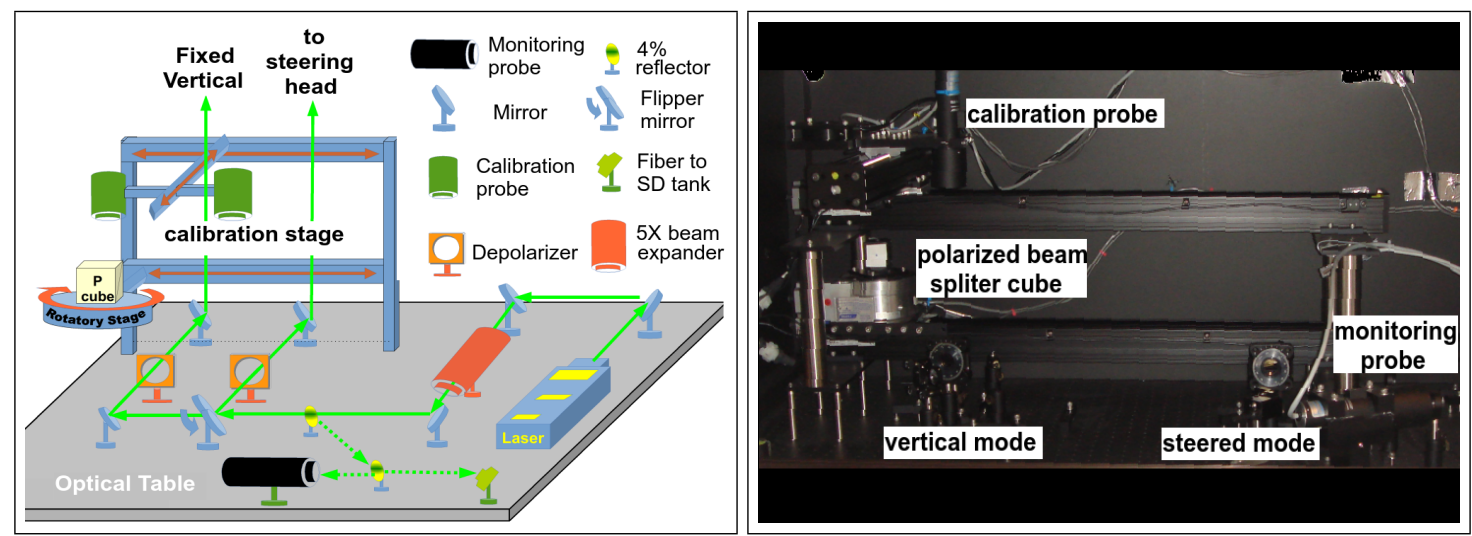

Figure 3: The calibration stage. (Left) A schematic diagram of the calibration system components on the optical table. (Right) The calibration system as fabricated.

Before the upgrade, absolute calibrations were performed manually at time intervals that ranged from a couple of months to one year. The available $C F$ measurements were linearly fit to provide a calibration function for different epochs. Epochs correspond to dates where the hardware was changed or the optics were cleaned. The calibration function is corrected to account for the amount of light captured by the probe that is not delivered into the sky, because of scattering off dust that had accumulated on the tilted mirror. The $C F$ distributions before and after the upgrade (Figure 4) demonstrate the improvement in the stability of the laser energy delivered to the sky and the frequency of the $C F$ measurements.
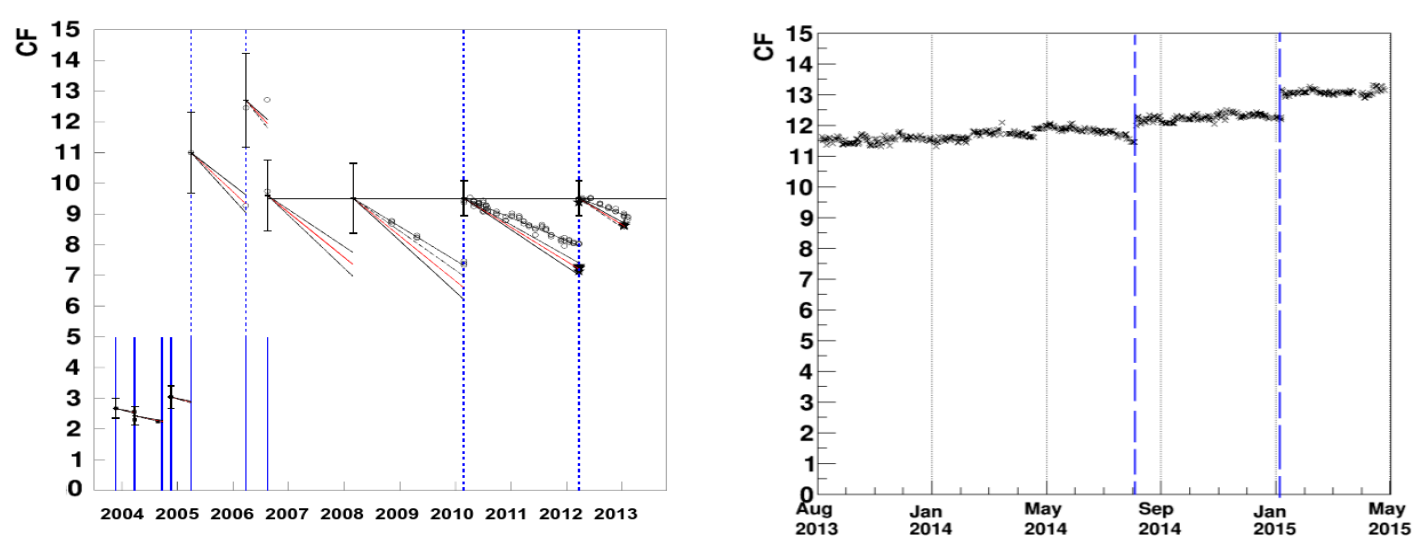

Figure 4: (Left, pre-ugrade) $C F$ are shown in circles. Black lines represent fit functions including corrections and blue lines represent epochs. (Right, post-upgrade) $C F$ are measured every day. Calibration functions and fits are no longer required.

A laser beam without net polarization is preferred because the atmospheric scattering is symmetric to the beam axis. To achieve this, the CLF uses polarization randomizers. The calibration 
system measures the net polarization, by rotating a polarization beam-splittering cube in front of the calibration probes, and by measuring the $C F$. The data points are fit to the function:

$$
P_{0}+\left(P_{1} \cdot \sin \left(\theta+P_{2}\right)\right)
$$

The energy variations due to polarization for 6 months of data are presented in Figure 5 (left). The fitting function is presented in Figure 5 (right) in polar coordinates and overlapping a circle of radius $P_{0}$ that represents a beam without polarization. The maximum deviation of the fitted line from the circle at angles facing the four FD sites is no larger than $1 \%$.
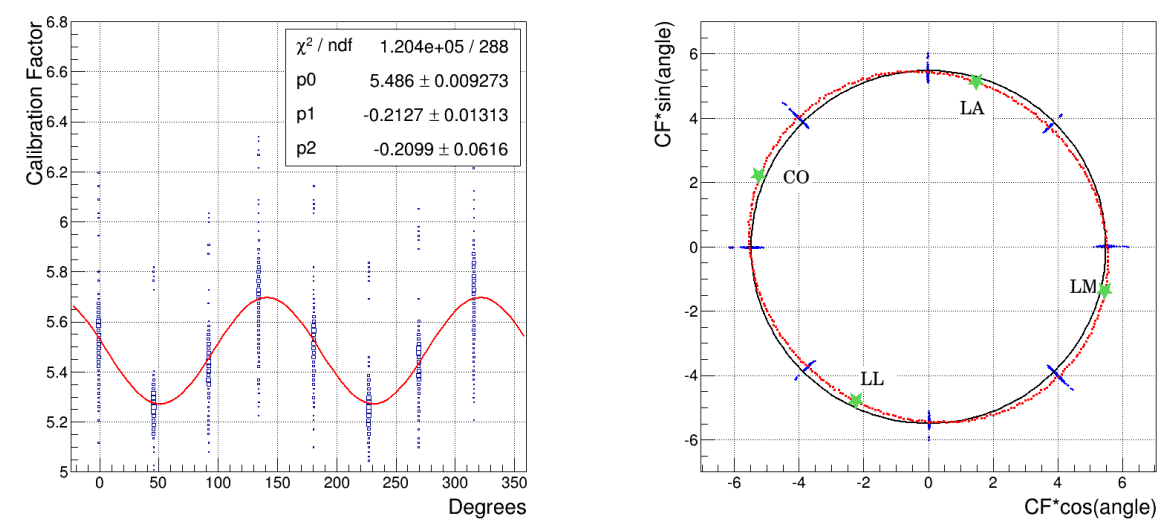

Figure 5: (Left) Six months of polarization calibration data. Blue points represent energy measurements at eight different rotation angles of the polarization analyzer. The red line represents the fit function. (Right) Data and fit line are presented in polar coordinates. The black circle of radius $P_{0}$ represents a beam without net polarization. The four green stars represent the directions toward the four FD sites.

\subsection{The Raman LIDAR}

The Raman LIDAR measures the back-scatter light from the laser to obtain $\tau(\mathrm{z}, \mathrm{t})$ independently [6]. The system has three channels: one for elastic scattering, one for Raman scattering off $\mathrm{N}_{2}$ molecules, and one for Raman scattering off water vapor (Figure 6).
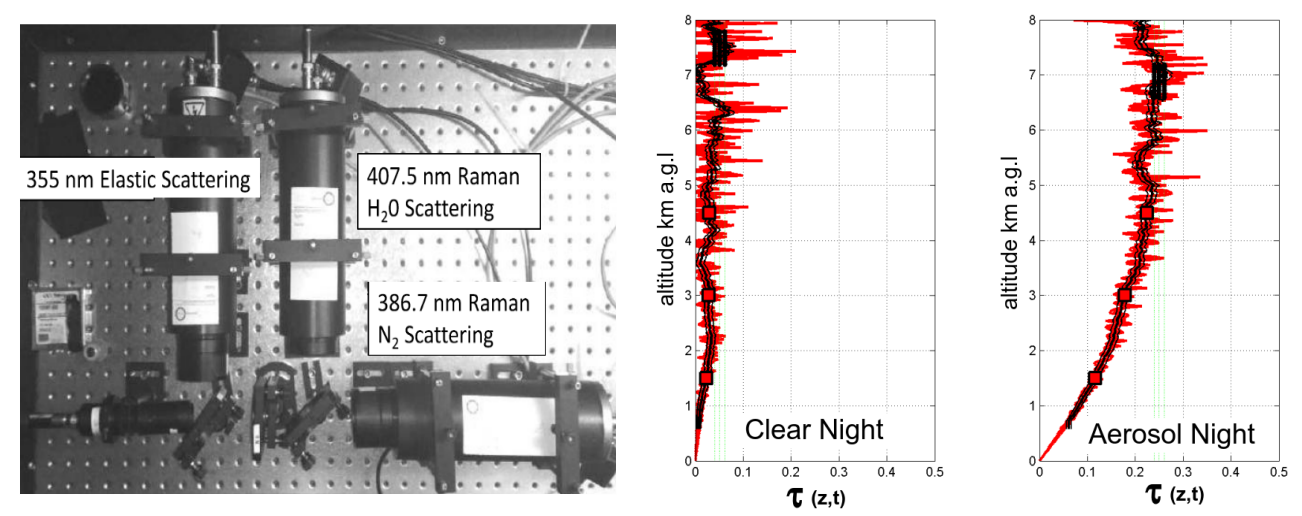

Figure 6: (Left) A picture of the Raman LIDAR optical components. (Right) Examples of hourly $\tau(\mathrm{z}, \mathrm{t})$ during a clear and a typical night with aerosols. 
The low cross section of Raman scattering requires a relatively long laser exposure to reduce statistical uncertainties. The Raman LIDAR runs take about 24 minutes. Shorter and longer runs can also be programmed. The Raman LIDAR has been running nightly before and after every FD shift since November 2013. To increase the number of $\tau(\mathrm{z}, \mathrm{t})$ measurements, an additional run was added in the middle of the FD shift beginning November 2014. During this additional Raman run, the FD bays overlooking the CLF are closed to avoid saturating the FD photomultipliers.

\section{Measurements and comparisons}

The $\tau(\mathrm{z}, \mathrm{t})$ profiles are calculated using a Data Normalized (DN) method. This method compares the FD response averaged over 200 laser shots (1 hour) with the FD response to 200 laser shots recorded during a clear, aerosol free, reference hour. Each average is normalized to the laser energy on a shot-by-shot basis. The ratio is used to obtain $\tau(\mathrm{z}, \mathrm{t})$ with a $400 \mathrm{~m}$ and a $1 \mathrm{~h}$ resolution. The reference hour is selected by scanning the highest hourly FD response during a one-year period. Most reference hours are found during winter periods. A FD response comparison between the 2014 reference hour (found on July 7th) and a typical aerosol-content-hour is shown in Figure 7 (left). The $\tau(\mathrm{z}, \mathrm{t})$ output of this comparison using the DN analysis is shown in Figure 7 (right).
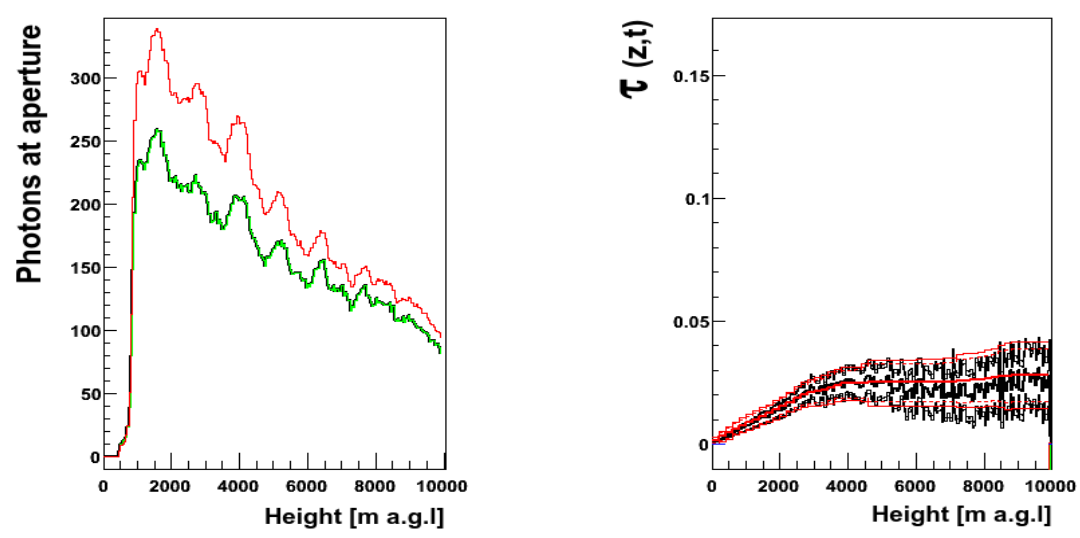

Figure 7: (Left) The green line represents the FD response in an hour with typical aerosol content and the red line represents extremely low aerosol content. (Right) The black traces represent $\tau(\mathrm{z}, \mathrm{t})$ and its uncertainties for an hour with typical aerosol content. The red traces are the fitted values.

The DN analysis uses the CLF for FD sites Los Leones, Los Morados and Coihueco, and uses the XLF for the FD site Loma Amarilla. This ensures that the scattered photons travel similar distances from the laser source to the FD sites. The following analysis will focus on results obtained with the upgraded CLF.

A comparison of $\tau(\mathrm{z}=3 \mathrm{~km})$ values obtained with the DN analysis at the same hour is presented in Figure 8 (left-center). The DN method presents good correlation for the FD sites Coihueco and Los Morados located at similar distances from the laser source. Similar results are found at different altitudes. Most outlying points are caused by scattering from clouds.

$\tau(\mathrm{z}, \mathrm{t})$ can be obtained using a second method called Laser Simulation (LS) analysis. This method compares the observed FD response to a set of simulated responses that are generated with 
different atmospheric parameters. The best fit to the actual FD response is selected. The LS method is used to fill in values of $\tau(\mathrm{z}, \mathrm{t})$ in the database that for various reasons are not obtained with the DN method. The details of the DN and the LS methods are described in [7]. A good correlation between the two methods using the CLF after the upgrade is observed (Figure 8(right)). The level of consistency between the two methods is the same as it was before the upgrade [8]. The $\tau(\mathrm{z}, \mathrm{t})$ values from both analyses are merged and registered into the observatory database for use in the reconstruction analysis of cosmic ray events [7].
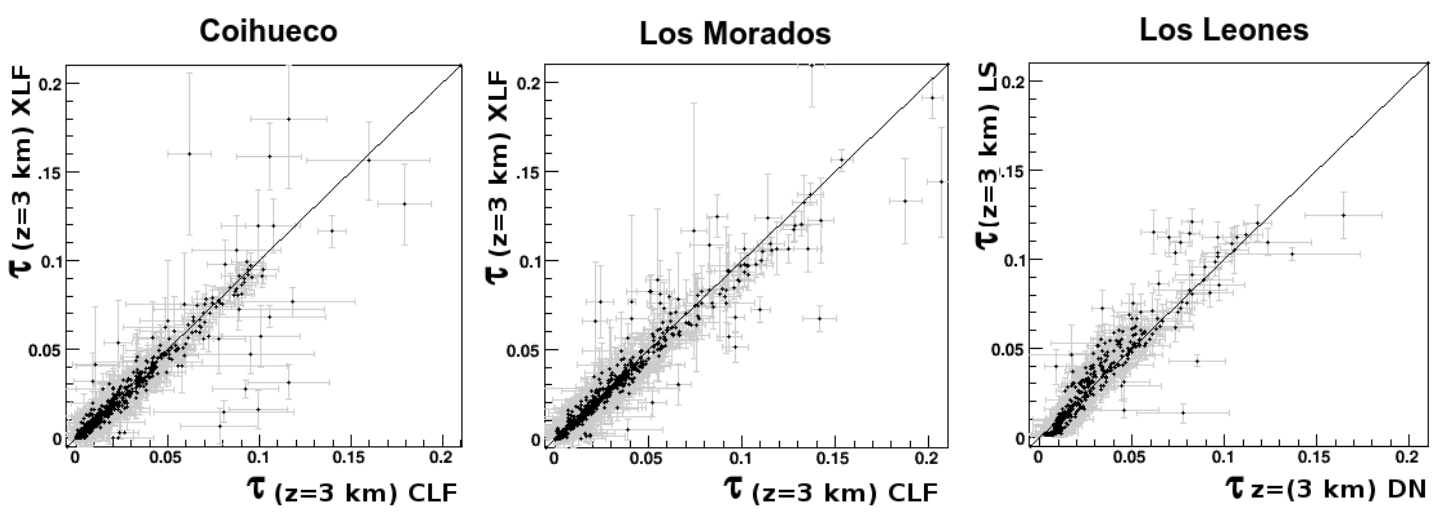

Figure 8: (Left and center) $\tau(\mathrm{z}=3 \mathrm{~km})$ correlations in 2014 between the CLF and XLF using the DN analysis. (Right) $\tau(\mathrm{z}=3 \mathrm{~km})$ correlations in 2014 between the DN and the LS analysis using the CLF.

A preliminary analysis of the Raman LIDAR measurements was used to obtain monthly averages of $\tau(\mathrm{z}, \mathrm{t})$ at different heights. An example of the $\tau(\mathrm{z}=3 \mathrm{~km})$ monthly average is presented in Figure 9. The data include one measurements of $\tau(\mathrm{z}, \mathrm{t})$ every night during 2014. Seasonal variations in the aerosol optical depth are observed.

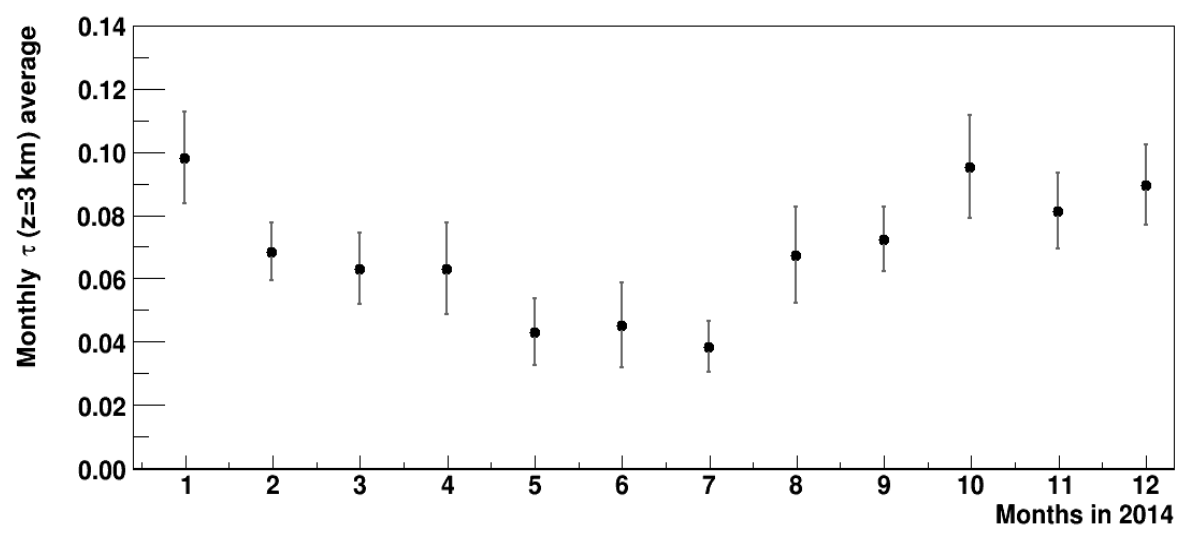

Figure 9: Preliminary monthly $\tau(\mathrm{z}=3 \mathrm{~km})$ averages measured with the Raman LIDAR. The error bars represent one sigma of statistical uncertainties.

Statistical uncertainties in the Raman LIDAR measurements are due to the small Raman cross section in combination with the limited laser exposure (24 minutes or less). Systematic uncertainties are being evaluated. This includes an examination of multiple scattering effects, an examination of the aerosol content during the reference hour and the use of a longer Raman run. 


\section{Conclusions}

The CLF has been upgraded to improve performance and add capabilities. The Raman LIDAR at the CLF is the first of its kind used for atmospheric measurements of $\tau(\mathrm{z}, \mathrm{t})$ in an UHECR experiment. The DN analysis provides consistent results for equidistant FD telescopes. The DN analysis results are consistent with the LS analysis. The Raman LIDAR has been used to measure $\tau(\mathrm{z}, \mathrm{t})$ every night for a full year. A preliminary analysis of the Raman LIDAR has been used to obtain averaged monthly values of $\tau(\mathrm{z}, \mathrm{t})$. Ongoing studies will continue to evaluate the systematic uncertainties and possible physics implications.

\section{References}

[1] The Pierre Auger Collaboration, A. Aab et al., The Pierre Auger Cosmic Ray Observatory, accepted for publication in Nucl. Instrum. Meth. A (2015); arXiv:1502.01323.

[2] The Pierre Auger Collaboration, J. Abraham et al., The Fluorescence Detector of the Pierre Auger Observatory, Nucl. Instrum. Meth. A 620 (2010) 227-251.

[3] B. Fick et al., The Central Laser Facility at the Pierre Auger Observatory, JINST 1, p1 1003 (2006), [astro-ph/0507334].

[4] The AIRFLY Collaboration, M. Ave et al., Measurement of the pressure dependence of air fluorescence emission induced by electrons, Astropart. Phys. 28 (2007) 41-57.

[5] L. Wiencke et al. for the Pierre Auger Collaboration, Atmospheric "Super Test Beam" for the Pierre Auger Observatory, in Proc. 32nd ICRC, Merida, Mexico, vol. 3 (2011), pp. 141-144.

[6] V. Rizi, A. Tonachini for the Pierre Auger Collaboration, M. Iarlori, G. Visconti, Atmospheric monitoring with LIDARs at the Pierre Auger Observatory, Eur. Phys. J. Plus (2012) 127:92.

[7] The Pierre Auger Collaboration, P. Abreu et al., Techniques for Measuring Aerosol Attenuation using the Central Laser Facility at the Pierre Auger Observatory, JINST 8, p04009 (2013); arXiv:1303.5576.

[8] L. Valore for the Pierre Auger Collaboration, Measuring Atmospheric Aerosol Attenuation at the Pierre Auger Observatory, in Proc. 33rd ICRC, Rio de Janeiro, Brazil (2013); arXiv:1307.5059. 